\title{
El neurodesarrollo a los dos años de vida de neonatos tratados en una unidad de cuidados intensivos neonatales
}

\author{
Luis Alberto Fernández Carrocera, ${ }^{1}$ Adriana Jonguitud Aguilar, ${ }^{1}$ \\ Eduardo Ortigosa Corona, ${ }^{2}$ René Humberto Barrera Reyes, ${ }^{1}$ \\ Carlos Martínez Cruz, ${ }^{1}$ María del Pilar Ibarra Reyes ${ }^{1}$ \\ y Lilían Rodríguez Pérez ${ }^{3}$
}

RESUMEN El objetivo principal de este estudio fue evaluar de forma prospectiva, a los 2 años de vida, el desarrollo de un grupo de neonatos tratados en la unidad de cuidados intensivos neonatales (UCIN) del Instituto Nacional de Perinatología de México. Se estudió desde el punto de vista neurológico, psicológico, auditivo, lingüístico, motor y neuromuscular a todos los neonatos nacidos entre el 1 de enero de 1992 y el 31 de diciembre de 1993 que hubieran ingresado a la UCIN y permanecido en ella 3 días o más. Se incluyó a 134 pacientes con una edad gestacional promedio de 32 semanas y un peso promedio al nacer de $1677 \mathrm{~g}$. De ellos, 75\% habían sido sometidos a ventilación mecánica, con una estancia hospitalaria promedio de 51 días. En el examen efectuado a los 2 años, 66,5\% de los niños fueron normales y 8,2\% tuvieron alteraciones graves. Se encontraron asociaciones significativas entre el estado neurológico y los días de ventilación artificial $(\mathrm{P}<0,0001)$, los días de estancia en la UCIN $(\mathrm{P}<0,000004)$ y la edad gestacional en semanas $(\mathrm{P}<0,03)$. No hubo ninguna asociación entre el sexo y el resultado de las valoraciones. En conclusión, los resultados obtenidos en este trabajo muestran una disminución de las alteraciones del neurodesarrollo en comparación con los resultados obtenidos en estudios similares hace 10 años.

Los adelantos recientes en las unidades de cuidados intensivos neonatales (UCIN) han cambiado los patrones de

\footnotetext{
1 Instituto Nacional de Perinatología, Departamento de Seguimiento Pediátrico, México D.F. Dirección postal: Instituto Nacional de Perinatología, Montes Urales 800, Col. Lomas Virreyes, 11000 México D.F., México.

2 Instituto Nacional de Perinatología, Departamento de Educación para la Reproducción, México D.F., México.

3 Instituto Nacional de Perinatología, Departamento de Psicología, México D.F., México.
}

mortalidad y morbilidad de los neonatos de riesgo elevado (1). La mortalidad ha declinado notablemente pero han aparecido alteraciones del neurodesarrollo, graves o sutiles, que han producido un aumento de las secuelas discapacitantes (2). Por lo tanto, ha surgido una controversia acerca de la atención de los pequeños que se encuentran en el límite de la viabilidad y que requieren procedimientos invasores caros por largo tiempo en las
UCIN, independientemente de su edad gestacional, peso, pronóstico y tipo de padecimiento $(3,4)$.

Se han publicado algunos trabajos sobre el desarrollo de los niños egresados de UCIN en términos de su salud general y desarrollo cognoscitivo, psicosocial y escolar (5-7). Estos estudios han mostrado que los recién nacidos pretérmino tienen mayor déficit cognoscitivo y dificultades de aprendizaje específicas y generales que conducen a 
falla escolar y a una gran utilización de recursos especiales para su educación (8). Estos niños también tienen problemas con la integración visual-motora, déficit de atención, peor salud general y menor desarrollo físico en comparación con controles normales (6).

En México, algunos estudios han evaluado las secuelas de audición, lenguaje, neurodesarrollo y estado de salud general de los neonatos egresados de UCIN y han encontrado una incidencia de secuelas similar a la notificada en la literatura médica. En diversas publicaciones se encontró que los procesos patológicos relacionados con secuelas del desarrollo fueron, en su mayor parte, trastornos del sistema nervioso central (9-20).

Desde la década de los sesenta, muchos recursos en tiempo y dinero se han dedicado a la atención aguda del recién nacido de alto riesgo, y ello ha producido una disminución notoria y constante de la mortalidad neonatal (21). Más sorprendente ha sido la supervivencia de los recién nacidos de muy bajo peso o con trastornos que antes se consideraban incompatibles con la vida $(9,22)$.

No obstante, los datos de mortalidad y morbilidad no reflejan por sí solos la complejidad del problema; un índice de sobrevida alto no significa que un elevado porcentaje de los sobrevivientes sea normal. Por ejemplo, es posible (con el apoyo ventilatorio intenso y prolongado) lograr índices bajos de mortalidad en neonatos que han sufrido hipoxia grave, hemorragia intracraneana o ambas, pero que después desarrollan secuelas neurológicas graves y deficiencias intelectuales. Esto sucedió cuando se utilizó de manera extensa la ventilación de recién nacidos de muy bajo peso ( $<1500 \mathrm{~g})$. Los índices de mortalidad (que para la década de los sesenta oscilaban entre 74 y $80 \%$ ) se redujeron a 45 ó $50 \%$ en los años setenta; sin embargo, los defectos neurológicos de los sobrevivientes fueron más serios en la última década y se asociaron con mayor frecuencia con secuelas neurológicas graves $(3,21)$.

Según informes más recientes, aunque preliminares, sobre la evolución de los recién nacidos de muy bajo peso tratados en centros de atención perinatal de alto riesgo, la mejor sobrevida en estos centros se acompaña también de un mayor porcentaje de sobrevivientes sin discapacidad (23-26). Por otra parte, aunque existen controversias en cuanto al género, algunos autores han encontrado mayor número de lesiones en el sexo masculino $(27,28)$. El objetivo principal de este estudio fue evaluar de forma prospectiva el desarrollo neurológico, a los 2 años de vida, de un grupo de neonatos tratados en una UCIN.

\section{MATERIALES Y MÉTODOS}

El Instituto Nacional de Perinatología de México inició hace 12 años el Programa de Seguimiento Pediátrico para Niños de Alto Riesgo. En él ingresan todos los neonatos que se consideran en alto riesgo de presentar secuelas neurológicas, como los recién nacidos con un peso menor de $1500 \mathrm{~g}$, edad gestacional menor de 34 semanas, hemorragia intracraneana, hidrocefalia, ventilación mecánica intermitente, asfixia grave, septicemia y displasia broncopulmonar. El programa tiene como propósito la detección presintomática de las discapacidades, para lo cual diversos especialistas realizan exámenes seriados del niño a fin de determinar si se está desarrollando normalmente.

En 1993 habían egresado 2600 niños del programa, principalmente de las unidades de cuidados intensivos e intermedios neonatales. La deserción fue de $20 \%$ y, de acuerdo con los estudios realizados por el servicio de trabajo social, las tres principales causas fueron la falta de recursos económicos familiares, la lejanía domiciliaria y el cambio de residencia a otro estado de la República Mexicana.

En este estudio se incluyó a todos los neonatos que ingresaron a la UCIN entre el 1 de enero de 1992 y el 31 de diciembre de 1993 por 3 días o más. Se excluyó a todos los pacientes que presentaron malformaciones congénitas mayores o que abandonaron el programa de seguimiento antes de los 2 años de vida.
Las variables en estudio fueron sexo, peso al nacer, edad gestacional (en semanas), morbilidad neonatal, utilización y días de ventilación artificial, días de estancia hospitalaria y resultados de la evaluación neurológica, psicológica, auditiva, del lenguaje y del tono muscular.

Para todos los pacientes incluidos en el estudio, se calculó la edad gestacional según la fecha de la última menstruación, siempre que fuera segura, o según el método de Capurro (29). Para todas las valoraciones se corrigió la edad gestacional a 37 semanas, lo cual es necesario en los neonatos prematuros para realizar los exámenes.

La evaluación neurológica se realizó con la exploración clínica de Corominas Beret (30). Los niños se clasificaron como normales cuando tuvieron buenos resultados en todos los indicadores de la prueba y como alterados cuando tuvieron resultados anormales en uno o más indicadores. Las alteraciones se clasificaron en leves (alteraciones del tono muscular y algunos signos blandos como hiperreflexia, desviación ocular inconstante, irritabilidad); moderadas (diplejía, hemiplejía o hipertonía) y graves (parálisis cerebral, retraso mental y crisis convulsivas).

Para la valoración psicológica se utilizó la prueba de Bayley, que contiene una escala mental y otra motora. Para ambas escalas se consideró normal un puntaje de 84 o más y alterado, uno de menos de 84 (31). La escala mental se dividió, a su vez, en las siguientes categorías: más de 115 puntos, coeficiente superior; de 114 a 85, promedio; de 84 a 70, inferior a la media; y de 69 o menos, retraso significativo.

Para la coordinación motora gruesa y fina se utilizó la valoración neuroconductual modificada por Helda Benavides, que clasifica de normales a los niños que tienen intactos los 10 indicadores; de retrasados, a los que tienen intactos 8 indicadores, y de alterados, a los que tienen intactos $7 \mathrm{o}$ menos (32). Se consideraron retrasados los que tenían una diferencia de 3 meses o menos en relación con la conducta esperada para su edad y alterados cuando la conducta para su edad tenía un retraso de más de 4 meses. 
Para evaluar el tono muscular se utilizó la valoración de Vojta (33), que lo clasifica de hipertónico, hipotónico, distónico y fluctuante.

La valoración audiológica se realizó en una cámara sonoamortiguada de $2 \mathrm{~m}^{2}$, con un audiómetro marca Maíco de dos canales, con calibración Ansi 1975 y linealidad de atenuación en un $\mathrm{KHz}$, en pasos de $10 \mathrm{db}( \pm 0,25 \mathrm{db})$. La audiometría se obtuvo por observación conductual en barrido de frecuencias y los niños se catalogaron como normales cuando la audiometría fue de 20 a $40 \mathrm{db}$; sospechosos, cuando fue de $41 \mathrm{a} 60 \mathrm{db}$; y anormales, cuando fue de $61 \mathrm{db}$ o más.

Para la valoración del lenguaje se utilizó un instrumento creado por el servicio de comunicación humana del Instituto Nacional de Perinatología, que cubre los aspectos fonológico, morfosintáctico y semántico, además de una prueba de articulación. En esta valoración los niños se clasificaron como normales cuando aprobaron los tres aspectos y alterados cuando presentaron problemas francos tanto en la comprensión lingüística como en la capacidad expresiva.

Los datos se analizaron por medio de frecuencias, medias, desviación estándar, diferencia de proporciones, prueba $\mathrm{F}$ del análisis de variabilidad (ANOVA), prueba de Kruskal-Wallis (KW) y riesgo relativo, con un nivel de confianza de $95 \%$.

\section{RESULTADOS}

Se estudió a 134 pacientes (67 femeninos y 67 masculinos) que reunieron los criterios de inclusión. En el cuadro 1 se muestran la edad gestacional, el peso al nacer, los días de ventilación artificial y los días de estancia.

Cuarenta y dos por ciento de los niños estudiados tuvieron un Apgar al minuto de 1 a 3 puntos; $33 \%$, de 4 a 6 , y $25 \%$, de 7 a 9 . A los 5 minutos $25 \%$ aún tenían un Apgar de 1 a 3 y el resto mejoró; de los que persistieron con Apgar bajo, 12\% presentaron algún tipo de alteración neurológica. Del total de la muestra, $85 \%$ fueron recién nacidos pretérmino; de estos, $28 \%$ fue-

CUADRO 1. Edad gestacional, peso al nacer, días de ventilación artificial y días de estancia, en una muestra de neonatos atendidos en una unidad de cuidados intensivos neonatales ( $n=134$ ). Instituto Nacional de Perinatología, México, D.F., 1992-1993

\begin{tabular}{lccc}
\hline \multicolumn{1}{c}{ Variables } & Promedio & DE & Recorrido \\
\hline Edad gestacional (semanas) $^{\mathrm{a}}$ & 32 & 2.9 & $26-41$ \\
Peso $(\mathrm{g})$ & 1677 & 728 & $575-3750$ \\
Días ventilación artificial $^{\mathrm{b}}$ & 9 & 12 & $1-80$ \\
Días de estancia en la UCINc $^{\mathrm{c}}$ & 51 & 34.6 & $3-180$ \\
\hline
\end{tabular}

a Neonatos pretérmino $=85 \%$.

${ }^{b}$ Neonatos que recibieron ventilación asistida $=75 \%$.

c Unidad de cuidados intensivos neonatales.

CUADRO 2. Edad gestacional, peso al nacer, días de ventilación artificial y días de estancia según sexo, en una muestra de neonatos atendidos en una unidad de cuidados intensivos neonatales. Instituto Nacional de Perinatología, México, D.F., 1992-1993

\begin{tabular}{lccc}
\hline \multicolumn{1}{c}{ Variables } & Masculino (promedio) & Femenino (promedio) & Significación \\
\hline Días de estancia en UCIN & 49,4 & 52,6 & n.s. $^{\mathrm{a}}$ \\
Edad gestacional (semanas) & 33,1 & 32,5 & n.s. $^{\text {b }}$ \\
Peso (g) & 1729 & 1626 & n.s. $^{\mathrm{a}}$ \\
Días de ventilación artificial & 9,1 & 8,9 & n.s. $^{\mathrm{b}}$ \\
\hline
\end{tabular}

a Análisis de varianza (ANOVA).

b Prueba de Kruskal-Wallis.

ron pequeños y $71 \%$ normales para su edad gestacional. Por otra parte, $75 \%$ de los neonatos recibieron ventilación artificial.

El sexo se examinó a la luz de las variables anteriores, pero las diferencias no fueron estadísticamente significativas (cuadro 2).

Con respecto a la morbilidad, resaltan en primer lugar los problemas infecciosos (septicemia y neumonías), seguidos de las alteraciones respiratorias propias del recién nacido pretérmino y posteriormente las complicaciones neurológicas (cuadro 3). Cabe aclarar que ningún trastorno se presentó de forma aislada, sino en combinación con otros.

El estado neurológico (normal o alterado) se examinó a la luz de algunas de las variables en estudio (cuadro 4). Por medio de la prueba de Kruskal-Wallis se encontraron asociaciones significativas con los días de ventilación artifical (KW=18,67; $P<0,0001)$, los días de estancia $(\mathrm{KW}=30,06 ; P<0,000004)$ y la edad gestacional en semanas $(\mathrm{KW}=$ 4,43; $P<0,03)$. El peso también mostró significancia estadística $($ ANOVA $=$
4,33; $P<0,03)$, pero no al examinarse según el sexo $\left(X^{2}=0,13\right)$.

Como se indica en el cuadro 5, el riesgo relativo de tener una alteración neurológica aumenta conforme disminuye el peso del recién nacido. Esta observación se reflejó en todos los rangos de peso al nacer.

CUADRO 3. Principales causas de morbilidad, en una muestra de neonatos atendidos en una unidad de cuidados intensivos neonatales. Instituto Nacional de Perinatología, México, D.F., 1992-1993

\begin{tabular}{lcc}
\hline \multicolumn{1}{c}{ Trastornos } & No. & $\%^{\mathrm{a}}$ \\
\hline Septicemia & 77 & 57 \\
Neumonía & 53 & 39 \\
$\quad$ Enfermedad de membrana & & \\
$\quad$ hialina & 52 & 38 \\
Displasia broncopulmonar & 51 & 37 \\
Apnea & 43 & 32 \\
$\quad$ Hemorragia intraventricular & 36 & 26 \\
Atelectasia & 29 & 21 \\
$\begin{array}{l}\text { Persistencia del conducto } \\
\text { arterioso }\end{array}$ & 26 & 19 \\
$\quad \begin{array}{l}\text { Taquipnea transitoria del } \\
\text { recién nacido }\end{array}$ & 19 & 14 \\
Hidrocefalia & 17 & 12 \\
\hline
\end{tabular}

a Representa el porcentaje de la muestra total. 
CUADRO 4. Resultados del examen neurológico a los 2 años de edad en función de algunas variables independientes $(n=134)$, en una muestra de neonatos atendidos en una unidad de cuidados intensivos neonatales (UCIN). Instituto Nacional de Perinatología, México, D.F., 1992-1993

\begin{tabular}{lccc}
\hline \multirow{2}{*}{ Variables } & \multicolumn{2}{c}{ Resultados del examen neurológico } \\
\cline { 2 - 4 } & Normal (promedio) & Alterado (promedio) & Valor $P$ \\
\hline Días de estancia en UCIN & 39,8 & 72,5 & $0,000004^{\mathrm{a}}$ \\
Edad gestacional (semanas) & 33,3 & 32,2 & $0,03^{\mathrm{a}}$ \\
Peso (g) & 1769 & 1495 & $0,03^{\mathrm{a}}$ \\
Días de ventilación artificial & 5,9 & 14,9 & $0,0001^{\mathrm{a}}$ \\
Sexo (No.) & & & n.s. $^{\mathrm{b}}$ \\
$\quad$ Masculino & 46 & 21 & \\
Femenino & 43 & 24 & \\
\hline
\end{tabular}

a Prueba de Kruskal-Wallis.

${ }^{b}\left(\chi^{2}\right)$.

CUADRO 5. Resultados del examen neurológico a los 2 años, según el peso al nacer, y riesgo relativo (RR) de alteración, en una muestra de neonatos atendidos en una unidad de cuidados intensivos neonatales. Instituto Nacional de Perinatología, México, D.F., 1992-1993

\begin{tabular}{|c|c|c|c|c|c|}
\hline \multirow{3}{*}{$\begin{array}{l}\text { Rangos de } \\
\text { peso al nacer (g) }\end{array}$} & \multicolumn{5}{|c|}{ Resultado del examen neurológico } \\
\hline & \multicolumn{2}{|c|}{ Normal } & \multicolumn{2}{|c|}{ Alterado } & \multirow[b]{2}{*}{$\mathrm{RR}$} \\
\hline & No. & $\%$ & No. & $\%$ & \\
\hline$\leq 1000$ & 7 & 41 & 10 & 59 & 1,70 \\
\hline $1001-1500$ & 38 & 66 & 20 & 34 & 1,28 \\
\hline $1501-2500$ & 30 & 73 & 11 & 27 & 1,20 \\
\hline$\geq 2501$ & 14 & 78 & 4 & 22 & - \\
\hline Total & 89 & 66 & 45 & 34 & \\
\hline
\end{tabular}

${ }^{a}$ Grupo de referencia.

De los 134 niños valorados neurológicamente, $67 \%$ resultaron normales y el resto de la muestra tuvo alteraciones $(Z=3,8 ; P<0,05)$. Se presentaron alteraciones leves en 22 pacientes, o $16 \%$ de toda la muestra; 12 niños (9\%) tuvieron alteraciones moderadas y 11 pacientes (8\%) tuvieron alteraciones graves.

La evaluación de Bayley se realizó en 93 pacientes. Se clasificaron como alterados $49 \%$ en la esfera mental y $48 \%$ en la psicomotora, pero la diferencia no fue significativa cuando se compararon con los normales. Tampoco dio resultados significativos el ANOVA al examinarse el rendimiento en función de la ventilación. No hubo una asociación significativa entre el sexo y el resultado de la valoración psicológica. De acuerdo a la clasificación mental de Bayley por puntaje, ningún niño tuvo un cociente mental superior; $50 \%$ tuvieron un cociente promedio; $22 \%$ lo tuvieron inferior a la media, y $28 \%$ presentaron un retraso grave.

La audición y el lenguaje se valoraron en 112 pacientes. De ellos, 80,3\% tuvieron la audición normal y la diferencia entre ellos y los alterados fue estadísticamente significativa $(Z=6,4$; $P<0,01)$ en relación con la audición. En 20 de los 22 alterados $(17,8 \%$ de toda la muestra) la lesión fue bilateral y de tipo neurosensorial profunda y en los otros dos casos fue unilateral (1,9\% de la muestra total). La valoración del lenguaje mostró alteración en 60,7\% de los niños; la diferencia con la proporción de los normales fue significativa $(Z=2,2 ; P<0,05)$. En $42,8 \%$ el tipo de lenguaje alterado fue el primario expresivo y en $17,9 \%$, el audiógeno. Ni la audición ni el lenguaje se mostraron significativamente diferentes en los dos sexos. Tampoco fue significativo el resultado del ANOVA entre los días de ventilación artificial y la audición.

Los resultados de la valoración de la coordinación motora y del tono muscular se presentan en el cuadro 6 .

\section{DISCUSIÓN}

Los neonatos gravemente enfermos difícilmente cursan con un trastorno único al que se pueda atribuir directamente la causa de la lesión o secuela neurológica; también influyen en el neurodesarrollo diversos factores socioeconómicos y ambientales. Aunque sería teóricamente posible buscar una asociación entre los diferentes trastornos neonatales y la presencia o el grado de lesión neurológica posterior, en este trabajo no se intentó ese análisis porque no pudo formarse un grupo control y porque ningún paciente presentó un trastorno aislado.

La edad gestacional y el peso al nacer son factores ya conocidos que se

CUADRO 6. Valoración de la coordinación motora gruesa y fina y del tono muscular, a los 2 años de edad, en una muestra de neonatos atendidos en una unidad de cuidados intensivos neonatales. Instituto Nacional de Perinatología, México, D.F., 1992-1993a

\begin{tabular}{lrr}
\hline \multicolumn{1}{c}{ Valoración } & No. & $\%$ \\
\hline $\begin{array}{l}\text { Coordinación motora } \\
\text { Gruesa }\end{array}$ & & \\
$\quad$ Normal & & \\
$\quad$ Retrasada & 93 & 79 \\
$\quad$ Alterada & 18 & 15 \\
Fina & \\
$\quad$ Normal & 7 & 6 \\
$\quad$ Retrasada & 89 & 75 \\
$\quad$ Alterada & 22 & 19 \\
& 7 & 6 \\
Tono muscular & & \\
$\quad$ Normalc & & \\
Alterado & \\
$\quad$ Hipertónico & 90 & 76 \\
$\quad$ Fluctuante & 28 & 34 \\
& 18 & 15.2 \\
a $Z=6,2 ; P<0,05$. & 8.4 \\
b $Z=5,6, P<0,05$. & 10 & \\
${ }^{c}$ Diferencia entre proporciones $(Z=5,7 ; P<0,05)$. &
\end{tabular}


asocian con los trastornos y enfermedades de los recién nacidos atendidos en las UCIN. Se trata básicamente de procesos infecciosos y respiratorios, así como de lesiones del sistema nervioso central ocasionadas por hemorragias intraventriculares.

Un alto porcentaje de los neonatos que ingresaron a la UCIN necesitaron ventilación artificial por más de una semana. Si se suma a una estancia hospitalaria prolongada, esto favorece aún más el tipo de morbilidad mencionada y aumenta el riesgo de presentar secuelas del desarrollo.

La asociación entre el peso al nacer y el resultado neurológico se hizo evidente al calcularse el riesgo relativo de sufrir una alteración, que fue casi dos veces mayor en neonatos de bajo peso, como también se documenta en otras publicaciones (4-6).

Si bien $67 \%$ de los niños fueron normales desde el punto de vista neurológico, 33\% presentaron algún tipo de alteración. En $75 \%$ de los casos esta fue leve o moderada y con frecuencia susceptibles de corrección (34); en el 25\% restante la alteración fue grave. Estos porcentajes coinciden con los de otros informes (35-38). Sin embargo, hay que tomar en cuenta que diferencias en las características de la muestra (peso, edad gestacional, año de nacimiento, tipo de terapia intensiva, etc.) no siempre permiten hacer comparaciones válidas entre los resultados de distintos estudios. Es de esperarse que estas cifras aún se puedan disminuir a medida que mejoran la tecnología y el conocimiento médico.

Mientras mas tecnología necesitó el neonato para sobrevivir, mayor fue su riesgo de sufrir lesiones neurológicas, como se pudo documentar cuando se examinó la asociación entre los resultados de la valoración neurológica por un lado y la ventilación asistida y los días estancia en la UCIN por el otro. Se encontró que era estadísticamente significativa la mayor duración de la estancia en la UCIN en aquellos niños que tuvieron una exploración neurológica alterada.

La proporción de alteraciones encontradas con la valoración psicoló- gica de Bayley fue similar para las dos escalas (mental y motora) y no se encontraron diferencias cuando se investigó su asociación con la duración de la ventilación artificial. En cambio, varios trabajos documentan una asociación entre los días que se recibió ventilación asistida y los trastornos del desarrollo, específicamente un cociente intelectual mediano o inferior y alteraciones motoras en grado variable. Los cocientes intelectuales observados en los niños incluidos en esta investigación concuerdan con los notificados en la literatura médica $(1,4,24,28,39,40)$.

La audición es un factor poco estudiado dentro de las UCIN, pero se han puesto de manifiesto algunas medidas terapéuticas que pueden lesionar el oído: la ventilación artificial, la utilización de aminoglucósidos y el uso de ciertos diuréticos como el furosemide $(19,20)$. Sin embargo, en este trabajo no se encontró ninguna asociación significativa con la duración de la ventilación. Aunque $96 \%$ de los pacientes recibieron aminoglucósidos (amikacina) durante 14 días a dosis de $7,5 \mathrm{mg} / \mathrm{Kg}$, no fue posible determinar los niveles séricos del antibiótico. Por otro lado es conocido el daño neurológico que ocasiona la hiperbilirrubinemia grave. En los 22 niños con alteración auditiva, el promedio de bilirrubina indirecta fue de 15,3 mg/dL (DE $\pm 4,7 \mathrm{mg} / \mathrm{dL})$.

En este estudio $20 \%$ de los niños presentaron alguna alteración auditiva, cifra que se encuentra dentro del recorrido notificado por otros autores (41-43). La observación cada vez más frecuente de fármacos potencialmente ototóxicos propició que en la UCIN del Instituto se revisaran las normas en cuanto a su dosis, vía de administración y tiempo de infusión y al uso de antibióticos alternativos, para tratar de prevenir las lesiones auditivas. Indudablemente, los factores sociales y ambientales tienen un efecto específico en la rehabilitación auditiva de estos niños. Muchos de los padres de los niños estudiados no tienen más de una educación media (secundaria) y son obreros o trabajadores asalariados con un nivel socioeconómico medio o bajo. Por lo tanto, la evolución y el progreso de todo niño hipoacúsico en la organización del lenguaje no se rigen por criterios uniformes y dependen más bien del grado de pérdida auditiva, las aptitudes del niño, la labor de los padres y educadores, así como el entorno en general. En nuestro estudio solo $40 \%$ de los niños con lesión auditiva grave tuvieron el beneficio de utilizar auxiliares auditivos; el resto aún no los han adquirido, principalmente por motivos económicos.

La hipertonía fue el patrón que predominó en los niños estudiados; sin embargo, el porcentaje es bajo en comparación con los resultados de Powls et al. (28). Esta diferencia podría explicarse por los programas de estimulación neuromotora de los que se vieron beneficiados todos los pacientes del programa de seguimiento.

Llama la atención que en este trabajo no hubo asociación significativa entre el sexo y la presencia de alteraciones neurológicas, ya que algunos autores sí han encontrado una asociación. Hille (27) encontró en neonatos masculinos un mayor riesgo de presentar problemas escolares, mientras que Powls encontró mayores alteraciones motoras en neonatos de sexo femenino con un peso al nacimiento menor de $1250 \mathrm{~g}$ (28). Este aspecto sigue siendo controversial en la literatura, pero el sexo apenas se ha investigado como factor de riesgo por sí solo $(27,28)$.

Por último, cabe subrayar que los resultados que se obtengan con los niños atendidos en las UCIN dependen de la participación conjunta de un equipo multidisciplinario y de la atención integral que reciban los niños no solo al nacer, sino durante todo un programa de seguimiento.

En conclusión, los resultados obtenidos en este trabajo muestran una disminución de las alteraciones del neurodesarrollo en niños atendidos en UCIN en comparación con las observadas hace 10 años o más. Sin embargo, la aparición de nuevos trastornos debe alertar al equipo de salud a la necesidad de determinar si sus métodos terapéuticos pudieran estar afectando al desarrollo neurológico de los niños y de modificarlos si fuese necesario. 


\section{REFERENCIAS}

1. Whyte HE, Fitshandinge PM, Shennan AT, Lennox K, Smith L, Lacy J. Extreme inmaturity: Outcome of 568 pregnancies of 23-26 weeks' gestation. Obstet Gynecol 1993;87(1):1-7.

2. Resnick MB, Stralka K, Carter RL, Anet M, Bucciarelli R, Furlough R, et al. Effects of birth weight and sociodemographic variables on mental development of neonatal intensive care unit survivors. Am J Obstet Gynecol 1990; 162(2):374-378.

3. Sommenfelt K, Ellertsen B, Markestad T. Personality and behaviour in eight-year-old, nonhandicapped children with birth weight under 1500 g. Acta Paediat 1993;82:723-728.

4. Saigal S, Rosenbaum P, Stoskppf B, Hoult L, Furlong W, Feeny D, Borrows E, Torrance G. Comprehensive assessment of the health status of extremely low bith weight children at eight years of age: Comparison with a reference group. J Pediatr 1994;125:411-417.

5. Saigal S, Szatmari P, Rosenbaum P, Campbell D, King S. Intellectual and functional status at school entry of children who weighed 1000 grams or less at birth: A regional perspective of births in the 1980s. J Pediatr 1990;116:409-416.

6. Saigal S, Szatmari $P$, Rosenbaum $P$, Campbell $D$, King S. Cognitive abilities and school performance of extremely low birth weight children and matched term control children at age 8 years: A regional study. J Pediatr 1991; $118 ; 751-760$.

7. McCormick MC, Brooks-Gunn J, Workman Daniels K. The health and developmental status of very low-birth-weight children at school age. JAMA 1992;267:2204-2208.

8. Hack M, Breslau N, Aram D, Weissman B, Klein N, Borowski C. The effect of very low birth weight and social risk on neurocognitive abilities at school age. J Dev Behav Pediatr 1992;13:412-420.

9. Fernández-Carrocera LA, Cano-González R, Ortigosa-Corona E, Barroso-Aguirre J, UdaetaMora E. Evolución neurológica y psicomotora a los dos años de vida de recién nacidos que fueron sometidos a ventilación asistida. Bol Med Hosp Infant Mex 1991;48(5):334-340.

10. Vázquez-Gómez $P$, Fernandez-Carrocera LA, Bravo-Cabrera Z, Venta-Sobero JA, MontielGarcía G, Lozano-González C. Secuelas de audición y lenguaje en sobrevivientes de una Unidad de Cuidados Intensivos Neonatales. Bol Med Hosp Infant Mex 1990;47(6):385-389.

11. Fernández-Carrocera LA, Barzola Barrientos A, Ortigosa-Corona E, Ibarra-Reyes P, Martínez-Cruz C, Rodríguez-Pérez L, et al. Neurodesarrollo al año de edad en neonatos con peso igual o menor a $1000 \mathrm{~g}$ al nacer. Bol Med Hosp Infant Mex 1994;51(10):643-649.

12. Fernández-Carrocera LA, Herrera-Torres R, Venta-Sobero JA, Venta-Sobero JA, IbarraReyes P, Rodríguez-Pérez L, et al. Seguimiento durante el primer año de vida, de neonatos con muy bajo peso al nacer. Bol Med Hosp Infant Mex 1991;48(5):326-332.

13. Fernández Carrocera LA, Turcio Cortázar E, Garza Morales S, Rodrígiuez-Pérez L, UdaetaMora E. Alteraciones del neurodesarrollo en el primer año de vida en neonatos que pe- saron 2000 gramos o menos al nacer. Neurología 1992;7(5):98-101.

14. Fernández-Carrocera LA, Ugartachea-Hernández JC, Lozano-González $\mathrm{CH}$. Seguimiento longitudinal del neonato prematuro. Rev Latin Perinat 1987;7:20-25.

15. Rodríguez-Balderrama I, Udaeta-Mora E, Cardiel-Marmolejo LE, Vargas-Quintal F, Fernández-Carrocera LA. Sobrevida en recién nacidos de muy bajo peso al nacer (menores de 1500 gramos) con relación a la ventilación mecánica convencional. Bol Med Hosp Infant Mex 1992;49(1):26-31.

16. Fernández-Carrocera LA, Vera-Olvera JJ, Udaeta-Mora E, Ibarra-Reyes P, CardonaPérez A, Garza-Morales S. Resultados antropométricos y del desarrollo neuromotor al primero y segundo años de vida en neonatos pretérmino con hidrocefalia posthemorrágica. Perinatol Reprod Hum 1992;6(2):77-81.

17. Fernández-Carrocera LA, Patiño-Felix F, Udaeta-Mora E, Garza-Morales S, IbarraReyes P, Rodríguez-Pérez L. Hemorragia subependimaria/intraventricular en neonatos pretérmino. Evolución del neurodesarrollo durante el primer año de vida. Bol Med Hosp Infant Mex 1993;50(4):241-248.

18. Fernández-Carrocera LA, Guerrero-Navarrete $\mathrm{MB}$, Udaeta-Mora $\mathrm{E}$, Ibarra-Reyes $\mathrm{P}$ Garza-Morales S. Hemorragia subependimaria intraventricular neonatal y alteraciones neuromotoras al año de vida. Rev Esp Pediatr 1992;40(1):19-22.

19. Martínez-Cruz C, Fernández-Carrocera LA, Ortigosa-Corona E, Garza Morales S, Poblano A. Disfunción auditiva en niños egresados de una unidad de cuidado intensivo neonatal. Rev Esp Pediatr 1996;52(2):123-129.

20. Martínez-Cruz C, Poblano A, Fernández-Carrocera LA, Garza-Morales S. Factores de riesgo para hipoacusia y hallazgos audiométricos en una población preescolar egresada de una unidad de cuidados intensivos neonatales. Salud Pública Mex 1995;37:205-210.

21. Avery Gordon B. Cap 21. En: Avery Gordon B. Neonatología 4. México: Ed. Panamericana; 1993. pp. 413-429.

22. Bishop GC, Lobo ML. Case study of a highrisk neonate failing to thrive post-extracorporeal membrane oxygenation and post-necrotizing enterocolitis. J Pediatr-Nurs 1994;9(3): 166-174.

23. Watt JM, Robertson CM, Grace MG. Early prognosis for ambulation of neonatal intensive care survivors with cerebral palsy. Dev Med Child Neurol 1989;31(6):766-773.

24. Goodman JT, Malizia KE, Durieux SA, Mac Murray B, Bernard P. Bayley developmental performance at two years of age of neonates at risk for hearing loss. Dev Med Child Neurol 1990;32(8):689-697.

25. Hadders Algra M, Towen BC. Body measurements, neurological and behavioral development in six-year-old children born pretern and/or small-for-gestational-age. Early Hum Dev 1990;27(1):1-13.

26. Saigal S, Feeny D, Furlong W, Rosenbaum P, Burrows E, Torrance G. Comparison of the health-related quality of life of extremely low birth weight children and a reference group of children at age eight years. J Pediatr 1994;125: 418-425.

27. Hille ETM, Den Ouden AL, Bauer L, Van den Oudenrijn C, Brand R, Verloove-Vanhorik SP. School performance at nine years of age in very premature and very low birth weight infants: Perinatal risk factors and predictors at five years of age. J Pediatr 1994;125: 426-434.

28. Powls A, Botting N, Cooke WR, Marlow N. Motor impairment in children 12 to 13 years old with a birthwight of less than $1250 \mathrm{~g}$. Arch Dis Child 1995;73:62-66.

29. Capurro H. Método clínico para diagnosticar la edad gestacional en el recién nacido [tesis doctoral]. Montevideo: Universidad de República; 1973.

30. Corominas Beret F. Exploración clínica en neuropediatría. 2a ed. Madrid: Publicaciones Médicas ESPASXS; 1982

31. Bayley N. Bayley. Scales of infant development. New York: Psychological Corporation; 1969.

32. Benavides-González H, Fernández-Carrocera LA, Venta-Sobero JA, Bravo-Cabrera Z, Ibarra-Reyes MP, Barroso-Aguirre J. Utilidad de una valoración neuroconductual de tamiz en el primer año de vida. Perinatol Reprod Hum 1989;3:193-198.

33. Vojta V. Die cerebralen Bewegungsstörungen in sauling-slater. Frühdiagnose und frütherapie. Stuttgart: Enke Verlag; 1984.

34. Amiel Tison C, Grenier A. Valoración neurológica del recién nacido. Barcelona: Toray Massón; 1981.

35. Tudehope D, Burns YR, Gray PH, Mohay HA, $\mathrm{O}^{\prime}$ Callaghan MJ, Rogers YM. Changing patterns of survival and outcome at 4 years of children who weighted 500-999 g at birth. J Pediatr Child Healt 1995;31:451-456.

36. Kroner J, Hjelt K, Nielsen JE, Kardorf UB, Verder $\mathrm{H}$. Neonatal mortality and late sequelae in infants with birth weight under $1501 \mathrm{~g}$ or gestational age under 31 weeks, primarily admitted to a central hospital. Ugeskr Laeger 1995;157:5707-5711.

37. Meberg A, Solberg R. Ventilator treatment at a subregional neonatal unit. Experiences form the Vestfold central hospital. Tidsskr Nor Laegeforen 1995;115:1724-1727.

38. Synnes AR, Ling EW, Whitfield MF, Mackinnon M, Lopes L, Wong G, et al. Perinatal outcomes of a large cohort of extremely low gestational age infants (twenty-three to twenty-eight completed weeks of gestation). J Pediatr 1994;125:952-960.

39. Robertson CM, Etches PC, Kyle JM. Eightyear school performance and growth of preterm, small for gestational age infants: A comparative study with subjets matched for birth weight or for gestational age. J Pediatr 1990;116(1):19-26.

40. Largo RH, Graf S, Kundu S, Hunziker U, Molinari L. Predicting developmental outcome at school age from infant test of normal, at-risk and retarded infants. Dev Med Child Neurol 1990;32(1):30-45. 
41. Bergman Y, Hirsch RP, Fria TJ, Shapiro SM, Holzman Y, Painto MJ. Cause of hearing loss in the high risk premature infant. J Pediatr 1985;106:95-101.

42. Uziel A, Piron JP. Evoked otoacoustic emissions from normal newborns and babies ad- mited to an intensive care baby unit. Acta Otolaryngol 1991;482(Suppl):85-91.

43. Kawashiro N, Tuchihashi N, Araki A, Koga K, Kawano T. Hearing loss following discharge from NICU. Nippon Jibiikoka Gakkai Kaiho 1994;97:1056-1061.
Manuscrito recibido el 7 de julio de 1997 y aprobado para publicación en versión revisada el 13 de agosto de 1998.

ABSTRACT The principal objective of this study was to evaluate, at 2 years of age, the neurological development of a group of children who had been treated in the neonatal intensive care unit (NICU) of the National Institute of Perinatology of Mexico. All the children born between 1 January 1992 and 31 December 1993 who had entered the NICU and stayed for 3 or more days were studied from the neurological, psychological, auditory, linguistic, motor, and neuromuscular standpoint. This group included 134 patients, who had had an average gestational age of 32 weeks and an average birthweight of $1677 \mathrm{~g}$. They had stayed in the hospital an average of 51 days, and $75 \%$ of them had undergone artificial respiration. In the examination done at age $2,66.5 \%$ of the children were normal and $8.2 \%$ had serious impairments. There were statistically significant associations between their neurological condition and the days of artificial respiration $(P<0.0001)$, the days spent in the NICU $(P<0.000004)$, and the gestational age in weeks $(P<0.03)$. There was no association between the children's sex and the results of the assessments. The study results showed a decrease in neural abnormalities in comparison with the results obtained in similar studies 10 years earlier. 